\title{
Oligopoly games with Local Monopolistic Approximation
}

\author{
Gian Italo Bischi ${ }^{\mathrm{a}, *}$, Ahmad K. Naimzada ${ }^{\mathrm{b}}$, Lucia Sbragia ${ }^{\mathrm{a}}$ \\ ${ }^{a}$ Department of Economics, University of Urbino, Italy \\ ${ }^{\mathrm{b}}$ Department of Economics, University Bicocca, Milan, Italy
}

Received 7 November 2004; received in revised form 29 June 2005; accepted 23 August 2005

Available online 2 October 2006

\begin{abstract}
We propose an oligopoly game where quantity setting firms have incomplete information about the demand function. At each time step they solve a profit maximization problem assuming a linear demand function and ignoring the effects of the competitors' outputs. Despite such a rough approximation, that we call "Local Monopolistic Approximation" (LMA), the repeated game may converge at a Nash equilibrium of the game played under the assumption of full information. An explicit form of the dynamical system that describes the time evolution of oligopoly games with LMA is given for arbitrary differentiable demand functions, provided that the cost functions are linear or quadratic. In the case of isoelastic demand, we show that the game based on LMA always converges to a Nash equilibrium. This result, compared with "best reply" dynamics, shows that in this particular case less information implies more stability.

(C) 2006 Elsevier B.V. All rights reserved.
\end{abstract}

JEL classification: L13; D83; C61; C62

Keywords: Oligopoly games; Bounded rationality; Subjective demand; Nash equilibrium; Dynamical systems; Stability

\section{Introduction}

The notion of Nash equilibrium in an oligopoly game is based on the assumption that each firm knows the market where it operates and knows what the other firms in the market decide to do. In particular, each firm is assumed to know the entire demand curve for the good it produces. It is more likely, however, that real firms only use some local estimate of the demand function, obtained through market experiments, when they compute their strategic variables as solutions

* Corresponding author. Present address: via Saffi n. 42, I-61029 Urbino, Italy. Fax: +39 0722305550.

E-mail address: bischi@uniurb.it (G.I. Bischi). 
to a profit maximization problem. Some authors use terms like "estimated" or "perceived" or "subjective" demand function, in order to say that the demand function that the firms use to solve their profit maximization problem is obtained through market experiments or by some "rule of thumb" (Baumol and Quandt, 1964; Silvestre, 1977; Bonanno and Zeeman, 1985).

Many authors have recently investigated the possible outcomes of repeated oligopoly games where the players have a misspecified knowledge of the demand function. Léonard and Nishimura (1999) examined discrete time dynamic duopolies and illustrated how the steady states (that are no longer Nash equilibria of the true game) change their stability properties as the result of the incorrect assessment of the demand function, the misspecification due to a multiplicative scale factor. Bischi et al. (2004a) propose a duopoly model where the players lack knowledge of the market demand and, different from the model of Léonard and Nishimura, the assumption of decreasing reaction functions is relaxed. This implies that new steady states (that are not Nash Equilibria) may be created when (one or both) players over- or underestimate the demand.

Interesting cases are obtained when an oligopoly game, repeatedly played by boundedly rational players who do not know the demand function, converges in the long run to a Nash equilibrium (i.e. to the same equilibrium that is reached in one-shot under full rationality). This may be seen as an evolutionary explanation of the outcome of a Nash equilibrium, and in the case of several Nash equilibria the repeated game may act as an equilibrium selection device. Of course, the more refined the decision-making process, the more expensive it is likely to be. Therefore, especially when a (single) decision is not of crucial importance, no more than an approximate solution may be justified. Some authors denote as "optimally imperfect decisions" the decisions based on simple and inexpensive computations, well suited for frequent repetition (on this point, see Baumol and Quandt).

This approach has been recently developed in a paper by Tuinstra (2004), where the subjective demand framework is used in a discrete-time price adjustment process. Following the seminal papers by Negishi (1961), Tuinstra assumes that, at each time step, price setting firms use subjective linear demand functions that depend only upon their own price and pass through the pricequantity combinations of the current state of the economy. Moreover, as in Silvestre, the slope of the subjective demand curve is assumed to coincide with the slope of the objective (generally nonlinear) demand curve. The firms observe the amount of the current price and they compute the slope of the true demand curve at that price. With this information they estimate a linear demand curve, and by using this estimate they set a new optimal price. In the case of linear cost functions, Tuinstra obtains an explicit discrete time dynamical system to describe the price adjustment process and investigates its dynamical properties. He also gives sufficient conditions for stability that involve cross-price effects and the curvature of the demand curve, and by using particular nonlinear demand functions associated with linear cost functions, he shows the occurrence of non-convergent trajectories and complicated dynamics.

In this paper we propose a similar adjustment mechanism for a repeated oligopoly game where quantity-setting firms solve a profit maximization problem by using a linear approximation of the demand function. As in Tuinstra (2004) this approximation is based on the estimate of the partial derivative of the demand function computed in the current state of the market and ignoring the presence of the competitors. No efforts are made by the players to learn the true (and generally nonlinear) demand function. ${ }^{1}$ Firms are just assumed to perform market experiments in a neighborhood of the current state of the market. It is assumed that through these experiments each firm

\footnotetext{
${ }^{1}$ A model with a learning mechanism has recently been proposed by Bischi et al. (2004b) that consider Cournot oligopolies where players know that the demand and cost functions are linear. Firms are assumed to know the cost
} 
obtains a correct estimate of the partial derivative of the inverse demand function with respect to its own quantity. This estimate is then used to obtain a linear approximation (and extrapolation) of the demand function without any guess about the influence of the competitors (i.e. a monopolistic approximation). However, even if the firms solve their profit maximization problems on the basis of such a rough approximation, which we call "Local Monopolistic Approximation" (LMA henceforth) the repeated game may converge at a Nash equilibrium of the true oligopoly game (i.e. the game played under the assumption of full information). In other words, the repeated game with LMA has the same (Nash) equilibria as the so called "best reply" dynamics, and it is interesting to compare the stability properties of a Nash equilibria under these two different adjustment mechanisms, based on different information sets. In order to make such a comparison, it is necessary to find suitable demand and cost functions that allow us to have an explicit discrete dynamical system. As we shall see, this is easily obtained for the LMA model by considering an arbitrary differentiable demand function, not only in the case of linear cost functions (as in the case of price adjustment considered by Tuinstra) but also in the case of quadratic cost functions. This is an important result, as quadratic costs are considered in several economic applications, for example in the harvesting of renewable resources (e.g., Clark, 1990; Szidarovszky and Okuguchi, 1998; Bischi et al., 2005). Instead, it is difficult to obtain explicit dynamical systems that represent best reply dynamics, as an explicit analytic expression of reaction functions is rarely found.

An exception is the Cournot oligopoly game with best reply obtained by using linear costs and an isoelastic demand function, as proposed by Puu (1991). In the case of homogeneous products and isoelastic demand function we can compare the stability of the Nash equilibrium under these two different kinds of profit maximizing output adjustment mechanism, based on LMA and best reply respectively. In fact, the properties of best reply dynamics of Cournot duopoly games has been extensively studied by Puu $(1991,1996)$ who showed that trajectories may not converge to the Cournot-Nash equilibrium and that complex trajectories are possible. In this paper we show that the repeated game based on LMA with an isoelastic demand function always converges to a Nash equilibrium. This implies that in this case adjustment mechanisms with LMA are more stable than the "best reply" dynamics, obtained under the assumption of full knowledge of the demand. In other words, in this case less information leads to more stability. However, as we shall see, the stability ranking between these two different adjustment mechanisms is not so immediate when we also consider the basins of attraction of the Nash equilibrium when it is stable under both dynamic processes. Moreover, the oscillatory (even chaotic) dynamics obtained under best reply may produce, on average, more profits than the stationary dynamics obtained under the adjustment with LMA. However, a repeated oligopoly game that converges to a Nash Equilibrium gives rise to a situation much more favorable for expectations formation.

The paper is organized as follows. In Section 2 we recall the setup and the notations of Cournot oligopoly games with best reply dynamics, and we briefly recall the results obtained by Puu (1991) for the Cournot duopoly with best reply and isoelastic demand function. In Section 3 we introduce the adjustment mechanism based on LMA, we discuss the information set required to perform it, and we give some general stability conditions. In Section 4 we study the existence and stability of a Nash equilibrium under LMA with an isoelastic demand function and linear cost functions, and we compare these results with those obtained under the best reply dynamics applied to the same economic context. In Section 5 we consider an aggregate model that describes the dynamics of

functions, but they misspecify the slope of the demand function, and they try to learn the true slope on the basis of the discrepancy they observe, at any repetition of the game, between the expected price and the realized one. 
the total output of the oligopoly system when homogeneous products, linear costs and isoelastic demand functions are considered, and we show that a higher number of firms leads to instability. In Section 6 we prove the stability of the Nash equilibrium under the LMA dynamics with isoelastic demand and quadratic costs. Section 7 concludes and indicates some possible directions and developments of future researches.

\section{Best reply dynamics}

Let us consider an industry where $n$ firms, indexed by $i=1, \ldots, n$, produce homogeneous products, with production levels $q_{i}, i=1, \ldots, n$, respectively. Strategic interaction arises because the price of the goods depends on the total output of the industry according to a given inverse demand function

$$
p=f(Q)
$$

where $Q=\sum_{i=1}^{n} q_{i}$ is the total output of the oligopoly market. If $C_{i}\left(q_{i}\right)$ denotes the cost function of producer $i$, then the profit at time period $t$ is

$$
\pi_{i}(t)=p(t) q_{i}(t)-C_{i}\left(q_{i}(t)\right) .
$$

The producers are assumed to be quantity setting, that is, at each time $t$ they decide the next-period productions $q_{i}(t+1)$ by maximizing the profit expected at the next period $t+1$ :

$$
q_{i}(t+1)=\arg \max _{q_{i}} \pi_{i}^{\mathrm{e}}(t+1)=\arg \max _{q_{i}}\left[p^{\mathrm{e}}(t+1) q_{i}-C_{i}\left(q_{i}\right)\right]
$$

where $p^{\mathrm{e}}(t+1)$ represents the price expected by player $i$ for period $(t+1)$. In the traditional Cournot game players are assumed to know the demand function, so each player $i$ expresses the expected price $p^{\mathrm{e}}(t+1)$ by using the inverse demand functions

$$
p^{\mathrm{e}}(t+1)=f\left(q_{i}(t+1)+q_{-i}^{\mathrm{e}}(t+1)\right)
$$

where $q_{-i}^{\mathrm{e}}(t+1)$ represents the output decisions of other players as expected by player $i$.

The Cournot optimization problem becomes

$$
q_{i}(t+1)=\arg \max _{q_{i}}\left[f\left(q_{i}+q_{-i}^{\mathrm{e}}(t+1)\right) q_{i}-C_{i}\left(q_{i}\right)\right] .
$$

In the simplest (and lucky) cases one can uniquely express $q_{i}$ as functions of $q_{-i}^{\mathrm{e}}$ :

$$
q_{i}(t+1)=r_{i}\left(q_{-i}^{\mathrm{e}}(t+1)\right)
$$

where $r_{i}$ are the reaction functions. The Nash equilibria correspond to the fixed points of the map (6), that is, they are located at the intersections of the reaction curves. If players correctly forecast the competitors' decisions, $q_{-i}^{\mathrm{e}}(t+1)=q_{-i}(t+1)$, then the Nash equilibria can be directly computed, in one-shot. Instead, in a bounded rationality setting, players may not know beforehand the competitors' choices, and consequently they formulate some reasonable forecast, on the basis of their information set. The simplest assumption, proposed by Cournot (1838), is that of naive expectations, $q_{-i}^{\mathrm{e}}(t+1)=q_{-i}(t)$, in which each firm expects that the production of the other firms will remain the same as in current period. ${ }^{2}$ Under this assumption (6) gives rise to

\footnotetext{
2 Other kinds of expectations mechanisms can be used, such as adaptive expectations, see for example Szidarovszky and Okuguchi (1988) and Bischi and Kopel (2001).
} 
a discrete-time dynamical system (Section 2)

$$
q_{i}(t+1)=r_{i}\left(q_{-i}(t)\right)
$$

Every Nash equilibrium is also an equilibrium of the best Reply dynamics, because the intersections of the reaction curves are the fixed points of (7). However, such equilibria are not reached in one shot. They may be reached asymptotically, in the long run, if they are stable under the best reply dynamics. This may be seen as an evolutionary explanation of the outcome of a Nash equilibrium, and in the case of several Nash equilibria the repeated game (7) may act as an equilibrium selection device. However, the dynamical system (7) may not converge to a Nash equilibrium, and it may exhibit asymptotic convergence to periodic or chaotic attractors (see e.g. Rand, 1978; Dana and Montrucchio, 1986; Puu, 1991, 1998; Bischi et al., 2000). In particular, Puu (1991) considered a Cournot duopoly game with an isoelastic demand (with unitary elasticity),

$$
p=f\left(q_{1}+q_{2}\right)=\frac{1}{q_{1}+q_{2}},
$$

together with linear cost functions $C_{i}=c_{i} q_{i}, i=1,2$. He proved that unimodal reaction functions are obtained, and the best reply dynamics can give rise to complex trajectories that do not converge to a Nash equilibrium. These reaction functions can be easily obtained by solving the optimization problem:

$$
q_{i}(t+1)=\arg \max _{q_{i}} \frac{q_{i}}{q_{i}+q_{-i}^{\mathrm{e}}(t+1)}-c_{i} q_{i},
$$

whose first order conditions become

$$
\frac{q_{-i}^{\mathrm{e}}(t+1)}{\left[q_{i}+q_{-i}^{\mathrm{e}}(t+1)\right]^{2}}-c_{i}=0,
$$

from which $q_{i}$ is obtained as a solution of a second degree algebraic equation, namely $q_{i}=$ $-q_{-i}^{\mathrm{e}} \pm \sqrt{q_{-i}^{\mathrm{e}} / c_{i}}$. Only the solution with " + " represents a maximum, hence the dynamical system obtained by Puu assuming Cournot expectations is given by

$$
\begin{aligned}
& q_{1}(t+1)=-q_{2}(t)+\sqrt{\frac{q_{2}(t)}{c_{1}}} \\
& q_{2}(t+1)=-q_{1}(t)+\sqrt{\frac{q_{1}(t)}{c_{2}}}
\end{aligned}
$$

A unique Nash equilibrium exists, given by

$$
\mathbf{q}^{*}=\left(q_{1}^{*} ; q_{2}^{*}\right)=\left(\frac{c_{2}}{\left(c_{1}+c_{2}\right)^{2}} ; \frac{c_{1}}{\left(c_{1}+c_{2}\right)^{2}}\right),
$$

whose stability properties are given by Puu (1991) in terms of the ratio between the marginal costs $c_{1} / c_{2}$. First of all, feasible (i.e. bounded and non negative) trajectories of the best reply dynamics are obtained provided that $c_{1} / c_{2} \in[4 / 25,25 / 4]=[0.16,6.25]$. Moreover, the Nash equilibrium (10) is stable if and only if $c_{1} / c_{2} \in(3-2 \sqrt{2}, 3+2 \sqrt{2}) \simeq(0.17,5.83)$. If $c_{1} / c_{2}$ exits this interval then the Nash equilibrium loses stability via a period doubling bifurcation. Indeed, Puu (1991) shows that if $c_{1} / c_{2}$ falls outside the interval $(3-2 \sqrt{2}, 3+2 \sqrt{2})$ then the asymptotic dynamics may converge at periodic cycles or even exhibit chaotic motion around the Nash equilibrium, as shown in Fig. 1a, where a chaotic trajectory is shown, together with the reaction curves, obtained 


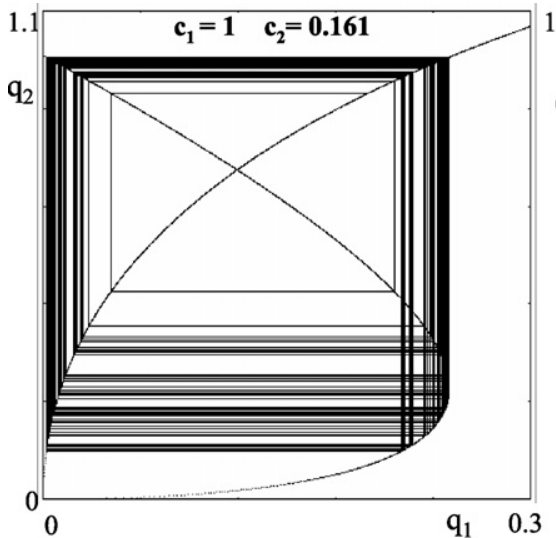

(a)



(b)

Fig. 1. Duopoly model with isoelastic demand (8), linear costs and best reply dynamics. (a) A chaotic trajectory obtained with parameters $c_{1}=1$ and $c_{2}=0.161$. (b) For the same model with $c_{1}=1$ and $c_{2}=0.7$, the white region represents the basin of attraction of the stable Nash Equilibrium, located at the intersection of the reaction curves, whereas the grey region represents the set of initial conditions that generate unfeasible trajectories.

with $c_{1}=1$ and $c_{2}=0.161$. In Fig. $1 \mathrm{~b}$, obtained with $c_{1}=1$ and $c_{2}=0.7$, the stable equilibrium (10) is shown, together with its basin of attraction, represented by the white region, whereas the grey region represents the set of initial conditions that generate unfeasible trajectories.

\section{The model with local and monopolistic approximation}

The best reply dynamics are obtained under the assumptions that, at each time, the firms have a global knowledge of the demand function and know their current production and the current production of the competitors as well, but they are not able to get a correct forecast of the competitors' choices for the next period. In this work we also consider another type of informational lacunae: we relax the assumption that each firm knows the demand function. However we assume that, through brief market experiments, at any time period each firm is able to get a correct estimate of the partial derivative

$$
f_{i}(t):=\frac{\partial f\left(q_{i}(t)+q_{-i}(t)\right)}{\partial q_{i}}=f^{\prime}(Q) .
$$

Then, each firm $i$ uses this estimate to obtain a "rule of thumb" computation of the expected price

$$
p^{\mathrm{e}}(t+1)=p(t)+f_{i}(t)\left(q_{i}(t+1)-q_{i}(t)\right)
$$

where $p_{i}(t)=f(Q(t))$ and $f_{i}(t)$ is defined in (11).

Of course, the approximation (12) is easier to be obtained than a global knowledge of the demand function (that involves values of $p$ or $q_{k}$ that may be very different from the current ones). Indeed, the estimate of $f_{i}(t)$ at time $t$ may be obtained by computing the effects of small price or quantity variations. For example, introducing at time $t$ a small output variation $\Delta q_{i}$, firm $i$ can compute

$$
\frac{f\left(q_{i}(t)+\Delta q_{i}+q_{-i}(t)\right)-f\left(q_{i}(t)+q_{-i}(t)\right)}{\Delta q_{i}},
$$


and we assume that this allows firm $i$ to get a correct estimate of $f_{i}(t)$. It is worth noting that an estimate of $f_{i}(t)$ can also be obtained through small price variations, such as

$$
\frac{\partial f(Q)}{\partial q_{i}}=\frac{\mathrm{d} f(Q)}{\mathrm{d} Q}=\left[\frac{\mathrm{d} Q(p)}{\mathrm{d} p}\right]^{-1},
$$

and therefore knowledge of the elasticity of market demand with respect to the market price is sufficient to determine (11). Indeed, price experiments are easier to perform than quantity experiments, and the price elasticity of the market demand at the current price usually belongs to the information set of the firms.

Notice that (12) is not a linear approximation of $f$, as firm $i$ neglects the influence of the competitor's production in the computation of the expected price. Of course, this is a very rough approximation. However, many authors claim that this is not far from reality; see for example Kirman (1975) on this point. Moreover, as we shall see below, even if the firms neglect the influence of competitors' outputs in the computation of the expected price, the dynamic process generated by such a repeated game may converge to the same equilibria as the best reply dynamics.

In fact, if the producer $i$ uses (12) to compute the expected price, then the first order conditions for the optimization problem (3) become

$$
\frac{\partial}{\partial q_{i}(t+1)}\left[q_{i}(t+1)\left(p(t)+f_{i}(t)\left(q_{i}(t+1)-q_{i}(t)\right)\right)-C_{i}\left(q_{i}(t+1)\right)\right]=0, \quad i=1, \ldots, n ;
$$

that is

$$
\left.p(t)+2 f_{i}(t) q_{i}(t+1)-f_{i}(t) q_{i}(t)\right)-C_{i}^{\prime}\left(q_{i}(t+1)\right)=0, \quad i=1, \ldots, n,
$$

where, again, $p(t)=f(Q(t)), f_{i}(t)$ is given by (11) and $C_{i}^{\prime}$ denoted the derivative of the cost function.

Notice that, in order to compute $q_{i}(t+1)$, at time $t$ firm $i$ needs the following information set:

(i1) Its current output $q_{i}(t)$;

(i2) The current price $p(t)$;

(i3) The partial derivative $f_{i}(t)$;

(i4) Its own cost function $C_{i}\left(q_{i}\right)$.

The following proposition states that even if the firms use such rough approximations of the inverse demand functions, the dynamic adjustment based on repeated production choices based on LMA has the same equilibria as the Cournot game with best replay. So, under suitable assumptions, the game with LMA may converge to a Nash equilibrium of the true game.

Proposition 1. The equilibria of the Cournot game (5) with best reply and perfect knowledge of the inverse demand function are steady states of the optimization problem with Local Monopolistic Approximation (15).

The proof of this proposition, given in Appendix in supplementary data available on the JEBO website, essentially shows that the first order conditions for the profit maximization problem (5) coincide with the steady state conditions obtained by setting $q_{i}(t+1)=q_{i}(t)$ in (15). Of course, the converse proposition, stating that an equilibrium of the dynamic process under LMA is also a Nash equilibrium of the true game (i.e. a profit maximum for every firm), would be interesting to prove, at least in specific cases. Of course, this also requires an analysis of second order conditions in (5). As we shall see, a relation between stable equilibria of the repeated game with LMA and 
Nash equilibria of the game under full information can be stated under suitable assumptions on cost functions.

Indeed, a study of the dynamic properties of the adjustment process (15), based on the Local Monopolistic Approximation of the demand function, is possible if the implicit Eq. (15) can be written in the form of an explicit discrete time dynamical system (if one can uniquely compute $q_{i}(t+1)$ from the knowledge the state variables at time $\left.t\right)$. This can be obtained if we consider suitable cost functions, such as the following two commonly used cost functions.

(i) Linear cost functions

$$
C_{i}=c_{i 0}+c_{i} q_{i}, \quad c_{i 0} \geqslant 0, c_{i}>0 .
$$

With this cost function we have $C_{i}^{\prime}\left(q_{i}(t+1)\right)=c_{i}$ and (15) gives

$$
q_{i}(t+1)=\frac{1}{2} q_{i}(t)-\frac{p(t)-c_{i}}{2 f_{i}(t)}, \quad i=1, \ldots, n
$$

where $p_{i}(t)=f(Q(t))$ and $f_{i}(t)$ is defined in (11).

(ii) Quadratic cost functions:

$$
C_{i}=c_{i 0}+c_{i} q_{i}^{2}, \quad c_{i 0} \geqslant 0, c_{i}>0 .
$$

With this cost function we have $C_{i}^{\prime}\left(q_{i}(t+1)\right)=2 c_{i} q_{i}(t+1)$, and (15) gives

$$
q_{i}(t+1)=\frac{q_{i}(t) f_{i}(t)-p(t)}{2\left[f_{i}(t)-c_{i}\right]}, \quad i=1, \ldots, n
$$

where, again, $p(t)=f(Q(t))$ and $f_{i}(t)$ is defined in (11).

Notice that in the price adjustment process proposed by Tuinstra only the case of linear cost functions gives rise to an explicit dynamical system. Instead, in our quantity-setting framework we can obtain an explicit expression of the dynamical system with quadratic costs. This may be important in several applications. To quote one important case, in fishery models the harvesting costs depend on the square of harvested quantity (e.g. Clark, 1990; Szidarovszky and Okuguchi, 1998; Bischi et al., 2005, where this quadratic cost function is derived from a "production function" of the Cobb-Douglas type with fishing effort and fish stock as the two inputs).

Thus, if we consider linear or quadratic cost functions and we assume several different kinds of nonlinear differentiable demand functions, the adjustment mechanisms (17) and (19) allow us to study several different dynamical systems obtained by using different nonlinear demand functions. It is interesting to obtain some ranking of the stability properties of the different models, based on the comparison of the regions of stability of the equilibria in the space of the parameters, or on the comparison of their basins of attraction, under different assumptions on the demand functions.

The following propositions, proved in Appendix in supplementary data, give sufficient conditions for the stability of a steady state under the two adjustment dynamics (17) and (19) respectively.

Proposition 2. Let $\mathbf{q}^{*}$ be an equilibrium for the oligopoly game defined by (5) with naive expectations and with the linear cost function (16), and let the inverse demand function $f(Q)$ be twice differentiable. Then $\mathbf{q}^{*}$ is a steady state of (17), and a sufficient condition for local stability of $\mathbf{q}^{*}$ under (17) is

$$
q_{i}^{*}\left|f^{\prime \prime}\left(Q^{*}\right)\right|+(n-1)\left|f^{\prime}\left(Q^{*}\right)+q_{i}^{*} f^{\prime \prime}\left(Q^{*}\right)\right| \leqslant 2\left|f^{\prime}\left(Q^{*}\right)\right| \text { for all } i=1, \ldots, n
$$

where $Q^{*}=\sum_{i=1}^{n} q_{i}^{*}$ is the total output at the equilibrium. 
Proposition 3. Let $\mathbf{q}^{*}$ be an equilibrium for the oligopoly game defined by (5) with naive expectations and with the quadratic cost function (18), and let the inverse demand function $f(Q)$ be twice differentiable. Then $\mathbf{q}^{*}$ is a steady state of (19), and a sufficient condition for local stability of $\mathbf{q}^{*}$ under (19) is

$$
q_{i}^{*}\left|f^{\prime \prime}\left(Q^{*}\right)\right|+(n-1)\left|f^{\prime}\left(Q^{*}\right)+q_{i}^{*} f^{\prime \prime}\left(Q^{*}\right)\right| \leqslant 2\left|f^{\prime}\left(Q^{*}\right)-c_{i}\right| \text { for all } i=1, \ldots, n
$$

where $Q^{*}=\sum_{i=1}^{n} q_{i}^{*}$ is the total output at the equilibrium.

These stability conditions are similar to the one given in Tuinstra for the price adjustment process with linear cost functions. Tuinstra provides an intuitive interpretation of such sufficient condition for stability terms of curvature of the demand functions, and he also shows that this sufficient condition also implies the second order condition for a profit maximum. This can be easily shown also in our model with linear costs. In fact, combining the necessary first order conditions for (5) $f(Q)+q_{i} f^{\prime}(Q)-c_{i}=0, i=1, \ldots, n$ (that coincide with the conditions for a steady state of (17)) with the second order conditions $q_{i} f^{\prime \prime}(Q)+2 f^{\prime}(Q) \leq 0, i=1, \ldots, n$, we get

$$
\frac{\left(f(Q)-c_{i}\right) f^{\prime \prime}(Q)}{\left[f^{\prime}(Q)\right]^{2}} \leq 2 .
$$

The sufficient condition for stability can be equivalently written as

$$
\frac{\left|f(Q)-c_{i}\right| f^{\prime \prime}(Q)}{\left[f^{\prime}(Q)\right]^{2}}+(n-1) \frac{\left|f^{\prime}(Q)+\left(c_{i}-f(Q)\right) f^{\prime \prime}(Q)\right|}{\left[f^{\prime}(Q)\right]^{2}} \leq 2
$$

because, from the first order conditions, $c_{i}-f(Q)=q_{i} f^{\prime}(Q)$, so it is evident that (22) is implied by (20). However, it is worth noticing the role of the number $n$ of firms in the oligopoly. In fact, a Nash equilibrium (i.e. a point $\mathbf{q}^{*}$ that represents a local profit maximum for all firms) may satisfy the sufficient condition for stability under the dynamics (17) only for a small number of firms. Indeed, in Section 5 we shall see that a Nash equilibrium, stable under best reply and under (17) with a low number of firms, can be destabilized for increasing $n$.

For the model with quadratic costs first order conditions for (5) are $f(Q)+q_{i} f^{\prime}(Q)-2 c_{i} q_{i}=$ $0, i=1, \ldots, n$ (that coincide with the conditions for a steady state of (19)); hence the second order conditions $q_{i} f^{\prime \prime}(Q)+2 f^{\prime}(Q)-2 c_{i} \leq 0, i=1, \ldots, n$, can be written as

$$
\frac{f(Q) f^{\prime \prime}(Q)}{\left[f^{\prime}(Q)-2 c_{i}\right]^{2}} \leq 2 \text {. }
$$

The sufficient condition for stability can be equivalently written as

$$
\frac{\left|f(Q)-c_{i} q_{i}\right| f^{\prime \prime}(Q)}{\left[f^{\prime}(Q)-c_{i}\right]^{2}}+(n-1) \frac{\left|f^{\prime}(Q)\left(c_{i}-f^{\prime}(Q)\right)+\left(f(Q)-c_{i} q_{i}\right) f^{\prime \prime}(Q)\right|}{\left[f^{\prime}(Q)-c_{i}\right]^{2}} \leq 2
$$

because, from the first order conditions, $f(Q)-q_{i} c_{i}=q_{i}\left(c_{i}-f^{\prime}(Q)\right)$, so a connection between (23) and (21) is not evident in this case. However, also in this case, the same arguments hold about the destabilizing role of $n$.

We end this section by stressing that, as in the case considered by Tuinstra, our statements cannot ensure that a stable equilibrium under LMA adjustment represents a profit maximum of the game under complete information, and they cannot ensure that a profit maximum of the game under complete information is necessarily a stable equilibrium. However, it is worth remarking 
that the players who at each time period solve their profit maximization problems according to the LMA always face a problem of maximization of a quadratic profit function; that is, at each time step they solve a standard problem of finding the unique global maximum of a concave (quadratic) perceived profit function. In other words, from their point of view (or, better, on the basis of their information set) they have no doubts that the solutions they find according to the first order conditions they compute are global maximum points for the profit functions they perceive.

In the following we shall focus our attention on the comparison between the stability properties of the Nash equilibria under the two different kinds of adjustment processes: the best reply dynamics and the dynamics with LMA. In order to make such a comparison, both of these dynamic adjustments must be expressed as explicit dynamical systems. As we have argued above, in the case of LMA this can be easily obtained for every differentiable demand function, provided that the cost functions are linear or quadratic. Instead, when using nonlinear demand functions, it is not easy to obtain an explicit form of the reaction functions. In fact, the optimization problem (5) may have a non-unique solution, and even in the case of uniqueness the expression of the corresponding reaction functions may be quite involved. As we have shown in Section 2, one of the fortunate cases where nonlinear reaction functions can be easily obtained, starting from a suitable nonlinear reaction function, is where an isoelastic demand function, associated with linear costs, is used.

In order to make such a comparison, in the following we consider oligopoly games with an isoelastic demand function.

\section{Cournot game with an isoelastic demand function and linear costs}

In this section we consider a duopoly model with the following isoelastic demand function,

$$
p=f\left(q_{1}, q_{2}\right)=\frac{1}{\left(q_{1}+q_{2}\right)^{\alpha}}, \quad \alpha>0,
$$

that for $\alpha=1$ reduces to the case studied by Puu (1991). The model (17) with $n=2$ and demand function (24) becomes a two dimensional dynamical system, defined by iterated map

$$
\begin{aligned}
& q_{1}(t+1)=\frac{1}{2} q_{1}(t)-\frac{1}{2 \alpha}\left(q_{1}(t)+q_{2}(t)\right)\left(c_{1}\left(q_{1}(t)+q_{2}(t)\right)^{\alpha}-1\right) \\
& q_{2}(t+1)=\frac{1}{2} q_{2}(t)-\frac{1}{2 \alpha}\left(q_{1}(t)+q_{2}(t)\right)\left(c_{1}\left(q_{1}(t)+q_{2}(t)\right)^{\alpha}-1\right) .
\end{aligned}
$$

The following proposition holds (the proof is in Appendix in supplementary data).

Proposition 4. If $\alpha<2$ the dynamical system (25) has a unique nonvanishing equilibrium, given by $q^{*}=\left(q_{1}^{*}, q_{2}^{*}\right)$, with

$$
\begin{aligned}
& q_{1}^{*}=\frac{1}{\alpha}\left(\frac{2-\alpha}{c_{1}+c_{2}}\right)^{(1 / \alpha)}\left(\frac{c_{2}+c_{1}(1-\alpha)}{c_{1}+c_{2}}\right) \\
& q_{2}^{*}=\frac{1}{\alpha}\left(\frac{2-\alpha}{c_{1}+c_{2}}\right)^{(1 / \alpha)}\left(\frac{c_{1}+c_{2}(1-\alpha)}{c_{1}+c_{2}}\right)
\end{aligned}
$$

which is positive if $\alpha>1-\left[\left(\min \left(c_{1}, c_{2}\right)\right) /\left(\max \left(c_{1}, c_{2}\right)\right)\right]$. This equilibrium is always locally asymptotically stable.

According to Proposition 1, a positive fixed point of (25) is also an equilibrium of the Cournot duopoly game with best reply. Of course, for $\alpha=1$ the positive equilibrium coincides of the 
positive equilibrium (10) of the duopoly model with best reply studied by Puu. Proposition 4 states that such equilibrium is always stable under the local monopolistic adjustment mechanism. This contrasts with the results on best reply dynamics discussed by Puu $(1991,1998)$ and described above.

So, in this particular case we can conclude that even if less informed players introduce rough approximations and correct their decision process every period, they converge to the optimal outcome (Nash equilibrium) for a wider range of parameters than in a game with players who know the true nonlinear demand and at each time step play the best reply. Indeed, in the case of isoelastic demand and linear costs, the convergence to the Nash equilibrium of the process with LMA is always ensured, whereas for certain sets of parameters the best reply dynamics does not converge to it. In other words, in this case we may guess that less information implies more stability.

However, we want to stress that this statement is obtained through a comparison of the stability region in the space of parameters $\left(c_{1}, c_{2}\right)$, in the sense that the Nash equilibrium $\mathbf{q}^{*}$ is stable in each points of the plane of the parameters $\left(c_{1}, c_{2}\right)$ for the model with LMA, whereas the stability only holds in the subset $c_{1} / c_{2} \in(3-2 \sqrt{2}, 3+2 \sqrt{2})$ in the case of best reply adjustment. Different conclusions may be obtained if we compare the basins of attraction. In fact, with cost parameters such that the Nash equilibrium is stable under both the adjustment mechanisms, larger basins of attraction can be observed for the model with best reply. This can be seen, for example, by a comparison of Figs. 2 and $1 \mathrm{~b}$, obtained with the same parameters $c_{1}=1$ and $c_{2}=0.7$, where Fig. 2 represents the basin of attraction (white region) of the stable Nash Equilibrium of the model (25) with LMA.

A further remark is related to the comparison between the long-run profits obtained by the players under the two different adjustment mechanisms (9) and (25). Of course, if both the repeated game with best reply and the one with Local Monopolistic Approximation converge to the same steady state $\mathbf{q}^{*}$, the long run average profits are the same. Instead, if the parameters are chosen in a range where the best reply dynamics exhibits bounded oscillations while the dynamics with LMA converge to the unique equilibrium, one may wonder which of the two

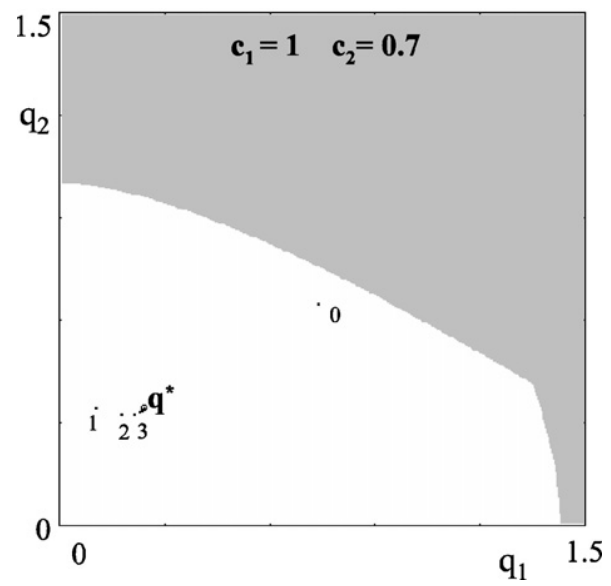

Fig. 2. Duopoly model with LMA dynamics, obtained with isoelastic demand (8) and linear costs. The parameter values are the same as in Fig. 1b. A typical trajectory that converges to the Nash equilibrium is also represented by dots labelled by $0,1, \ldots$. 

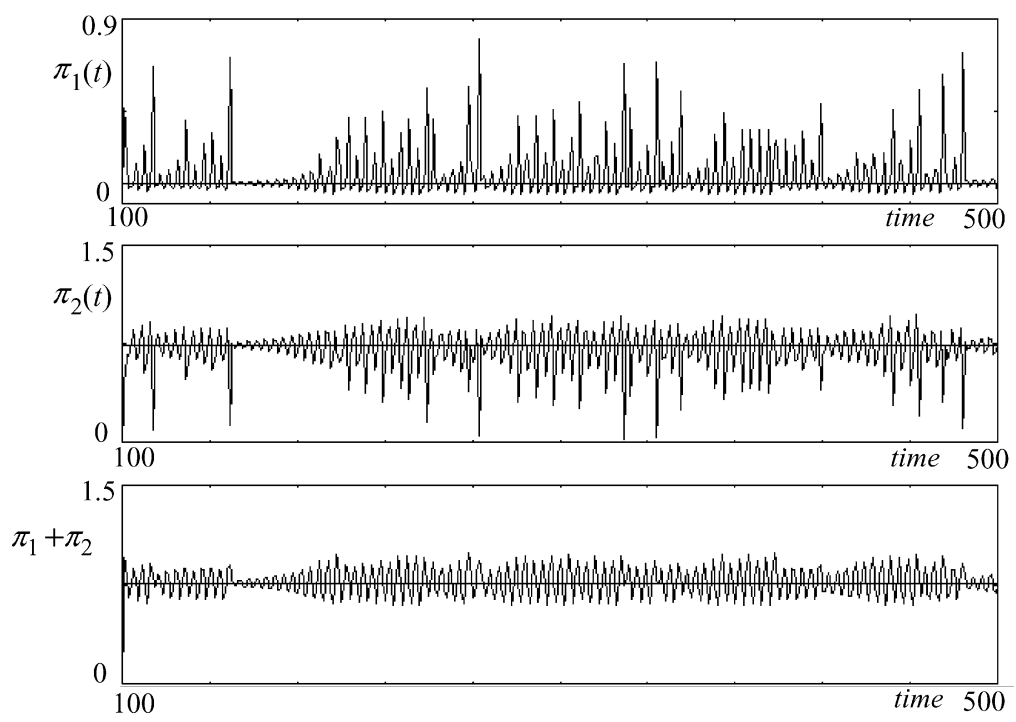

Fig. 3. Numerical computatons of individual profits $\pi_{1}(t)$ and $\pi_{2}(t)$ and total profit $\pi_{1}(t)+\pi_{2}(t)$ along a trajectory of the model with best reply (9) (the oscillatory ti,e pattern) and the model with LMA (25) (the horizontal lines), in the time range [100, 500], with parameters $\alpha=1, c_{1}=1, c_{2}=0.16$.

situations is more profitable on the average. An answer to this question is not easy, as stressed in several recent papers (see e.g. Huang, 1995; Matsumoto, 2003; Matsumoto and Nonaka, 2004, 2005). We performed some numerical computations of profits and their time averages in the long run behavior of the two dynamic adjustments with parameters $\alpha=1, c_{1}=1, c_{2}=0.16$. With this set of parameters the best reply exhibits chaotic oscillations whereas the dynamics with LMA converge to the steady state dynamics (10). Fig. 3 shows the time series of profits $\pi_{1}(t)$ and $\pi_{2}(t)$ computed along a generic trajectory of (9) compared with the profits computed according to the asymptotic dynamics of (25), that is, the constant profits computed at the steady state, $\pi_{1}^{*}=c_{2} /\left(c_{1}+c_{2}\right)^{2} \simeq 0.02$ and $\pi_{2}^{*}=c_{1} /\left(c_{1}+c_{2}\right)^{2} \simeq 0.74$. It can be seen that in this case the chaotic time pattern obtained under (9) is more profitable for player 1 , characterized by a higher cost, and less profitable for player 2 . Indeed, the average profits, computed along the time interval $[100,500]$, are $\bar{\pi}_{1} \simeq 0.07$ and $\bar{\pi}_{2} \simeq 0.71$. In the lowest graph in Fig. 3 the total profits $\pi_{1}(t)+\pi_{2}(t)$ are represented: the average total profit under LMA dynamics is $\pi_{1}^{*}+\pi_{2}^{*}(t) \simeq 0.76$ and the one under best reply dynamics is $\bar{\pi}_{1}+\bar{\pi}_{2} \simeq 0.78$ (i.e. the chaotic dynamics under best reply are slightly more profitable in this case). These results cannot be used to obtain any general statement; they are only given here to show that a comparison of long-run profits is not trivial in general.

In any case, more stability is preferable if the economic agents have to make forecasts about the future states of the economy.

\section{Case of isoelastic demand, linear costs and $\boldsymbol{n}$ players}

A question that is often discussed in the literature on oligopoly games concerns the effect of the number of players on stability. In general this issue is not an easy task, because an increase in the number of players implies an increase in the dimensions of the dynamical system. To obtain 
some insight into this question, let us consider the model (17)

$$
q_{i}(t+1)=\frac{1}{2} q_{i}(t)+\frac{c_{i}-f(Q(t))}{2 f^{\prime}(Q(t))}, \quad i=1, \ldots, n,
$$

being $f_{i}=f^{\prime}(Q(t))$ for each $i$. This $n$-dimensional dynamical system in the dynamic variables $q_{i}$ can be reduced to a one-dimensional dynamical system in the total quantity $Q(t)$ by summing up the Eqs. (26)

$$
Q(t+1)=\frac{1}{2} Q(t)+\frac{\gamma-n f(Q(t)}{2 f^{\prime}(Q(t))}
$$

where $\gamma=\sum_{i=1}^{n} c_{i}$. The dynamic equation (27) of the aggregate production includes the number of players $n \in \mathbb{N}$ as a parameter. Therefore, we can investigate the effects of this parameter on the dynamics of the global production.

It is trivial to see that if $\left(q_{1}^{*}, \ldots, q_{n}^{*}\right)$ is a steady state of the disaggregated dynamical system (26), then $Q^{*}=\sum_{i=1}^{n} q_{i}^{*}$ is a steady state of the aggregated dynamical system (27). In particular, if $\left(q_{1}^{*}, \ldots, q_{n}^{*}\right)$ is a Nash equilibrium, then it is a fixed point of (26) and consequently it corresponds to a fixed point of (27). However, the converse is not true in general because a fixed point $Q^{*}$ of (27) can correspond to several different arrangements of $\left(q_{1}, \ldots, q_{n}\right)$, that do not correspond to fixed points of (26).

We now consider the model (17) with $n$ firms and homogeneous products in the case of isoelastic demand function

$$
p=f(Q)=\frac{1}{Q} \text {. }
$$

Puu (1996), Agiza (1998) and Agliari et al. (2000) consider some particular cases with 3 or 4 competitors that repeatedly play the oligopoly game according to the best reply dynamics, given by the dynamic equations

$$
q_{i}(t+1)=\frac{1}{c_{i}} \sqrt{\sum_{j=1, j \neq i}^{n} q_{j}}-\sum_{j=1, j \neq i}^{n} q_{j}, \quad i=1, \ldots, n,
$$

and show several kinds of dynamic situations where the convergence to a Nash equilibrium does not occur. Indeed, quite complex dynamic scenarios may arise, characterized by periodic, quasiperiodic or chaotic motion.

If we assume LMA the dynamical system (26) with $n$ players and inverse demand function (28) becomes

$$
q_{i}(t+1)=\frac{1}{2}\left[q_{i}(t)+Q(t)-c_{i} Q^{2}(t)\right], \quad i=1, \ldots, n
$$

and the one-dimensional map (27) that describes the time evolution of the aggregated output $Q(t)$ becomes

$$
Q(t+1)=\frac{1}{2}[1+n-\gamma Q(t)] Q(t)
$$

where $\gamma=\sum_{i=1}^{n} c_{i}$. This is a quadratic one-dimensional map, topologically conjugate to the standard logistic map $x(t+1)=\mu x(t)(1-x(t))$ through the linear homeomorphism $Q=x(1+$ 
$n) / \gamma$ and with the parameters related by

$$
\mu=\frac{1+n}{2} \text {. }
$$

The time evolution of the aggregated production can be deduced from the well known properties of the logistic map (see e.g. Devaney, 1989). In particular, we are interested in the role of the integer parameter $n$.

First of all, we notice that the dynamics of (31) converge to the positive steady state $Q^{*}=(1+n-2) / \gamma$ provided that $n \leqslant 5$, corresponding to the well known condition $\mu \leqslant 3$. The convergence is monotone if $n \leqslant 3$, whereas it exhibits damped oscillations if $4 \leqslant n \leqslant 5$. With 6 players we have $\mu=3.5$; hence we have stable oscillations of period 4 being $\mu>$ $1+\sqrt{6}$. The case of 7 competitors produces fully developed chaos, as it corresponds with $\mu=4$.

Hence, stability is obtained for a limited number of oligopolists, namely $n \leqslant 5$, and instability occurs as the number of players increases.

\section{Duopoly with isoelastic demand and quadratic costs}

In this section we consider the duopoly model with isoelastic demand function (8) and quadratic costs (18). The best reply dynamics cannot be expressed by a simple dynamical system. In fact, the profit of player $i$ is $\pi_{i}=q_{i} /\left(q_{1}+q_{2}\right)^{2}-c_{i m}-c_{i} q_{i}^{2}$, and the first order conditions for profit maximization give rise to third degree algebraic equations. For example, the condition for the reaction function of player 1 becomes

$$
2 c_{1} q_{1}^{3}+4 c_{1} q_{2} q_{1}^{2}+2 c_{1} q_{2}^{2} q_{1}-q_{2}=0 .
$$

So, even if it is easy to see that a unique positive solution $q_{1}=r_{1}\left(q_{2}\right)$ exists, its expression is not easily handled. Instead, if we consider the dynamics with LMA (19) with isoelastic demand (8) and $n=2$, a simple two-dimensional dynamical system can be represented by the following two-dimensional iterated map:

$$
\begin{aligned}
& q_{1}(t+1)=\frac{2 q_{1}(t)+q_{2}(t)}{2\left(1+c_{1}\left(q_{1}(t)+q_{2}(t)\right)^{2}\right)} \\
& q_{2}(t+1)=\frac{q_{1}(t)+2 q_{2}(t)}{2\left(1+c_{2}\left(q_{1}(t)+q_{2}(t)\right)^{2}\right)} .
\end{aligned}
$$

The following proposition holds.

Proposition 5. The dynamical system (32) has a unique nonvanishing equilibrium, given by $\mathbf{q}^{*}=\left(q_{1}^{*}, q_{2}^{*}\right)$, with

$$
\begin{aligned}
& q_{1}^{*}=\frac{\sqrt{c_{2}}}{\sqrt{c_{1}}+\sqrt{c_{2}}} \frac{1}{\sqrt{2 \sqrt{c_{1} c_{2}}}} \\
& q_{2}^{*}=\frac{\sqrt{c_{1}}}{\sqrt{c_{1}}+\sqrt{c_{2}}} \frac{1}{\sqrt{2 \sqrt{c_{1} c_{2}}}} .
\end{aligned}
$$

This equilibrium is always locally asymptotically stable.

The proof of this proposition is given in Appendix in supplementary data.

According to Proposition $1, \mathbf{q}^{*}$ is located at the intersection of the reaction functions. As we do not know an explicit expression of the reaction functions, it is impossible to study its stability 
under best reply. However, such equilibrium reveals a strong stability under LMA. Simulations show that even the basins of attraction are large if compared with the case of the model with LMA and linear costs.

\section{Summary and further developments}

In this paper we have proposed a repeated oligopoly game where we assumed that the players do not know the demand function of the market in which they operate. Due to this lack of information, at each time step they solve profit maximization problems by using a linear approximation of the demand function, based on the local estimate of the partial derivative of the inverse demand with respect to their own outputs. Moreover, each player neglects the outputs of the competitors. A similar adjustment mechanism has been recently proposed by Tuinstra for a price adjustment process, and an explicit dynamical system is obtained for any differentiable demand function provided that cost functions are linear. Instead, our quantity-setting Local Monopolistic Approximation (LMA) gives rise to explicit discrete-time dynamical systems for linear or quadratic cost functions.

This kind of dynamic adjustment process has the same equilibria as the so-called best reply dynamics, obtained under the assumption of full knowledge of the demand function. Sufficient conditions are given for the stability of the equilibria under dynamics with LMA, and in the case of linear cost functions such conditions are shown to imply that the equilibria represent profit maximizing solutions. However, profit maximizing equilibria may be unstable both under best reply and under dynamics with LMA, especially when the number of players is increased.

As the oligopoly game obtained under the assumption of LMA has the same equilibria of the best reply dynamics (i.e. Nash Equilibria) we tried to compare the stability properties of such equilibria under these two different kinds of dynamic adjustments. This comparison has been performed by using one of the simplest nonlinear demand functions, the isoelastic one. In fact, this is one of the lucky cases where the reaction functions can be analytically computed (Puu, 1991) and consequently the dynamical system that gives the best reply dynamics can be explicitly written. The results obtained for this particular example show that adjustment processes based on LMA are more stable than best reply dynamics, even if characterized by a lower degree of information and rationality. So in the case we have analyzed, we obtain the intriguing statement that "less information leads to more stability". Furthermore, we have shown that an even stronger stability result is obtained when quadratic costs are considered.

In the case of an isoelastic demand function and linear costs we also analyzed the effects of increasing the number of competitors in the market, and we showed that more players may lead to periodic and chaotic motion.

As the dynamic adjustment mechanism proposed in this paper allows us to obtain an explicit dynamical system for any arbitrary nonlinear and differentiable demand function, provided that the cost functions are linear or quadratic, a plethora of dynamic models can be studied to compare stability and the dynamic properties under several economic assumptions about nonlinear demand functions that characterize the economy. In particular, the robustness of the statement "less information implies more stability", given in this paper on the basis of the example with an isoelastic demand function, can be analyzed by considering other examples obtained with different kinds of demand functions. Indeed, the driving force of the result may be the fact that, due to the linear approximation of a convex nonlinear demand function, firms always underestimate the price for 
given quantities, which leads to lower production quantities compared to the case of complete information. Hence, upward jumps are dampened and downward jumps become more pronounced. Of course, these properties are no longer true with different kinds of nonlinear demand functions, so one should investigate more deeply to understand, for the statement given above, how crucial the assumption that demand is isoelastic really is. Unfortunately, since it is generally difficult to find explicit dynamic equations for the best reply dynamics in the presence of nonlinear demand functions, it is not so easy to verify this result for other examples.

Moreover, through numerical investigations, we have argued that a comparison between the two adjustment mechanisms merits more in-depth investigation, for example the basins of attraction should be compared, as well as profits and welfare along the trajectories. We have only given some numerical computations for these topics.

The two different dynamic adjustment processes engaged in by the players, according to their information set, suggest an explicit modelling of time evolution of the two different strategies under evolutionary pressure. One may investigate an evolutionary game with firms meeting each other in pairs every period in order to play the Cournot duopoly game. A certain fraction of the population of firms produces according to the LMA, whereas the rest produces according to the best reply. These fractions change over time as a function of the realized profits generated by the different adjustment mechanisms, according to a replicator dynamics or by the switching mechanism proposed by Brock and Hommes (1997); see also Droste et al. (2002). Of course, the adjustment process characterized by more information should include an extra cost. An important question is whether one kind of adjustment will drive out the other one. Our example suggests that the answer is not trivial, as a strategy with less information may produce better performance than one characterized by more information. This is in contrast to the usual assumptions made in economic theory. A second important insight provided by the paper is the fact that an increase in the number of firms may lead to local instability, as is illustrated by the example in Section 5 .

Another remark about future work that may be done in the framework of oligopoly games with LMA concerns the presence of denominator in the maps (17) and (19). Indeed, some interesting dynamic phenomena can be expected, related to the peculiar properties of maps with a denominator that vanishes in a zero-measure subset of the state space, as stressed in Bischi et al. (1999, 2003).

\section{Acknowledgments}

The authors thank the participants to the Third International Conference on Nonlinear Economic Dynamics, Tokyo 2004, as well as two anonymous referees. This work has been performed within the activities of the research project "Le interazioni fra settore ittico e ambiente", financed by Italian Ministry for Agriculture, under the Joint Research Grant (0382): "Reconsideration of economic dynamics from a new perspective of nonlinear theory", Chuo University, Japan, and within the activity of the national research project "Nonlinear models in economics and finance: interactions, complexity and forecasting", MIUR, Italy.

\section{Appendix A. Supplementary data}

Supplementary data associated with this article can be found, in the online version, at doi:10.1016/j.jebo.2005.08.006. 


\section{References}

Agiza, H.N., 1998. Explicit stability zones for Cournot Game with 3 and 4 competitors. Chaos, Solitons \& Fractals 9 , 1955-1966.

Agliari, A., Gardini, L., Puu, T., 2000. The dynamics of a triopoly Cournot game. Chaos, Solitons \& Fractals 11, 25312560.

Baumol, W.J., Quandt, R.E., 1964. Rules of thumb and optimally imperfect decisions. The American Economic Review $54,23-46$.

Bischi, G.I., Chiarella, C., Kopel, M., 2004a. The long run outcomes and global dynamics of a duopoly game with misspecified demand functions. International Game Theory Review 6, 343-380.

Bischi, G.I., Gardini, L., Mira, C., 1999. Plane maps with denominator. Part I: some generic properties. International Journal of Bifurcation and Chaos 9, 119-153.

Bischi, G.I., Gardini, L., Mira, C., 2003. Plane maps with denominator. Part II: noninvertible maps with simple focal points. International Journal of Bifurcation and Chaos 13, 2253-2277.

Bischi, G.I., Kopel, M., 2001. Equilibrium selection in a nonlinear duopoly game with adaptive expectations. Journal of Economic Behavior and Organization 46, 73-100.

Bischi, G.I., Kopel, M., Szidarovszky, F., 2005. Expectation-stock dynamics in multi-agent fisheries. Annals of Operations Research 137, 299-329.

Bischi, G.I., Mammana, C., Gardini, L., 2000. Multistability and cyclic attractors in duopoly games. Chaos, Solitons \& Fractals 11, 543-564.

Bischi, G.I., Sbragia, L., Szidarovszky, F., 2004b. Learning the demand function in a repeated Cournot oligopoly game. Mimeo, University of Urbino.

Bonanno, G., Zeeman, C., 1985. Limited knowledge of demand and oligopoly equilibria. Journal of Economic Theory 35, 276-283.

Brock, W.A., Hommes, C.H., 1997. A rational route to randomness. Econometrica 65, 1059-1095.

Clark, C.W., 1990. Mathematical Bioeconomics—The Optimal Management of Renewable Resources, second ed. John Wiley \& Sons, New York.

Cournot, A., 1838. Recherches sur les principes matematiques de la theorie de la richesse. Hachette, Paris.

Dana, R.A., Montrucchio, L., 1986. Dynamic complexity in duopoly games. Journal of Economic Theory 40, 40-56.

Devaney, R.L., 1989. An Introduction to Chaotic Dynamical Systems. The Benjamin/Cummings Publishing Co., Menlo Park.

Droste, E., Hommes, C.H., Tuinstra, J., 2002. Endogenous fluctuations under evolutionary pressure in Cournot competition. Games and Economic Behavior 40, 232-269.

Kirman, A.P., 1975. Learning by firms about demand conditions. In: Day, R.H., Groves, T. (Eds.), Adaptive Economic Models. Academic Press, New York, pp. 137-156.

Huang, W., 1995. Caution implies profit. Journal of Economic Behavior and Organization 27, 257-277.

Léonard, D., Nishimura, K., 1999. Nonlinear dynamics in the Cournot model without full information. Annals of Operations Research 89, 165-173.

Matsumoto, A., 2003. Let it be: chaotic price instability can be beneficial. Chaos, Solitons \& Fractals 18, 745-758.

Matsumoto, A., Nonaka, Y., 2004. Profitable in a duopoly market with asymmetric production externality. Studies in Regional Sciences 35, 1-16.

Matsumoto, Nonaka, Y., 2005. Profitable and non-profitable chaos in a Cournot duopoly market. Mimeo, Chuo University, Tokyo.

Negishi, T., 1961. Monopolistic competition and general equilibrium. Review of Economic Studies 27, $136-139$.

Puu, T., 1991. Chaos in duopoly pricing. Chaos, Solitons \& Fractals 1, 573-581.

Puu, T., 1996. Complex dynamics with three oligopolists. Chaos, Solitons \& Fractals 7, 2075-2081.

Puu, T., 1998. The chaotic duopolists revisited. Journal of Economic Behavior and Organization 33, 385-394.

Rand, D., 1978. Exotic phenomena in games and duopoly models. Journal of Mathematical Economics 5, $173-184$.

Silvestre, J., 1977. A model of general equilibrium with monopolistic behavior. Journal of Economic Theory 16, 425442.

Szidarovszky, F., Okuguchi, K., 1988. A linear oligopoly model with adaptive expectations: stability reconsidered. Journal of Economics 48, 79-82.

Szidarovszky, F., Okuguchi, K., 1998. An Oligopoly model of commercial fishing. Seoul Journal of Economics 11, $321-330$

Tuinstra, J., 2004. A price adjustment process in a model of monopolistic competition. International Game Theory Review 6, 417-442. 


\section{Further Readings}

Atkinson, K.E., 1989. An Introduction to Numerical Analysis. John Wiley \& Sons, New York. Medio, A., Lines, M., 2001. Nonlinear Dynamics. Cambridge University Press. 